805-820, 1981.

[5] H. Mader, "Electrical properties of bulk-barrier diodes," $I E E E$ Trans. Electron Devices, vol. ED-29, no. 11, pp. 1766-1771, Nov. 1982.

[6] J. M. Shannon, "A majority-carrier camel diode," Appl. Whys. Lett. vol. 35, p. 63, 1979.

[7] E. Spenke, "p-n-Übergänge" in Halbleiter Elektronik, Bc.. 5. Berlin: Springer-Verlag, 1979.

[8] W. L. Engl, "Device modeling," Proc. IEEE, vol. 71, no. 1, pp. 10-16, Jan. 1983.

[9] D. M. Caughey and R. E. Thomas, "Carrier mobilities in silicon empirically related to doping and field," Proc. IEEE, vol. 55 , pp. 2192-2193, 1967.

[10] M. Takagi, K. Nakajama, and H. Kamioka, "Improvement of shallow base transistor technology by using a doped poly-silicon diffusion source," Japan. Soc. Appl. Phys. Supp., vol. 42, p. 101, 1978.

[11] M. A. Green and R. B. Godfrey, "Super-gain silicon MIS he:erojunction emitter transistors," IEEE Electron Device Lett., vol. EDL-4, no. 7, pp. 225-227, July 1983.
[12] C. H. Séquin and M. F. Tompsett, Charge transfer devices. New York: Academic, 1975.

[13] J.-I. Nishizawa, T. Terasaki, and J. Shibata, "Fieldeffect transistor versus analog transistor (static induction transistor), "IEEE Trans. Electron Devices, vol. ED-22, pp. 185-197, Apr. 1975.

[14] P. Plotko and B. Wilamowski, "Interpretation of exponentialtype drain characteristics of the static induction transistor," Solid-State Electron., vol. 23, pp. 693-694, 1980.

[15] J.-I. Nishizawa, T. Ohmi, and H.-L. Chen, "Analysis of static characteristics of a bipolar-mode SIT (BSIT)," IEEE Trans. Electron Devices, vol. ED-29, nol. 8, pp. 1233-1244, Aug. 1982.

[16] E. O. Johnson, "The insulated-gate field-effect transistor-A bipolar transistor in disguise," $R C A R e v$., vol. 34 , pp. $80-94$, Mar. 1973.

[17] A. Wieder, "Emitter effects in shallow bipolar devices-Measurements and consequences," IEEE Trans. Electron Devices, vol. ED-27, pp. 1402-1408, Aug. 1980.

[18] J. M. C. Stork and J. D. Plummer, "Small-geometry depleted base bipolar transistors (BSIT)-VLSI devices?," IEEE Trans. Electron Devices, vol. ED-28, pp. 1354-1363, Nov. 1981.

\title{
Small-Signal Charge Transfer Inefficiency Experiments Explained by the McWhorter Interface State Model
}

\author{
RENÉ G. M. PENNING DE VRIES AND HANS WALLINGA, MEMbER, IEEE
}

\begin{abstract}
The small-signal charge transfer inefficiency (SCTI) of a surface-channel CCD has been studied. The experimentally observed behavior of the SCTI could not be explained by the conventional interface state model. Using the McWhorter model for the interface states, which assumes a distribution of the states in the oxide perpendicular to the interface, an excellent agreement between theory and experiment is obtained. Essentially, this is due to the fact that in the McWho:ter model a spectrum of capture cross sections is associated with each enetgy level. No evidence for the edge effect has been found in devices with a channel width above $50 \mu \mathrm{m}$.
\end{abstract}

\section{INTRODUCTION}

$\mathrm{T}$ HE CHARGE transfer inefficiency (CTI) of surface-channel CCD's has been the subject of many theoretical and experimental studies. After the pioneering work of Carnes and Kosonocky [1], extensive studies about the influence of interface states on the CTI have been published by Tomps stt [2] and Mohsen et al. [3]. In the latter two papers, it vras

Manuscript received March 13, 1984; revised May 30,1984. This work was part of a program of the Foundation for Fundamental Research on Matter and was supported by the Foundation for Technical Researsh, Technical Science Branch, Division of the Netherlands Organization for the Advancement of Pure Research.

The authors are with the Twente University of Technology, 7500 AE Enschede, The Netherlands. concluded that the CTI of a SCCD operated with a bias charge (fat zero) and with a sufficiently low clock frequency to avoid limited transfer time effects is caused mainly by interface states situated at the parallel edges of the channel. This has been called edge effect [2]. By its very nature, the contribution of the edge effect to the CTI is inversely proportional to the channel width [3].

Various aspects of the CTI theory as presented in [2] and [3] have been confirmed by experiments. Kosonocky and Carnes [4] showed that the CTI has a tendency to increase with decreasing channel width, while Broderson et al. [5] found that the charge loss in the central gate area can be eliminated completely by the use of a sufficiently large bias charge. Klar et al. [6], however, could not clarify their experiments with the existing theory and they suggested that interface states in the perpendicular edge regions are mainly responsible for the CTI in CCD's with a large channel width. Only recently, Cheng and Viswanathan [7] presented data that could be described in terms of the theory as developed in [3]. However, the transfer time dependence of their model was not verified experimentally.

In all the experimental studies use was made of the so-called periodic pulse technique [5], or strongly related techniques, where the response of a CCD on a step signal is determined in 
the time domain. Because the size of the step signal is usually a considerable fraction of the full well capacity, an important parameter for the CTI, namely the amount of stored charge, is heavily changed during the measurement.

An approach which circumvents this disadvantage is based on the determination of the transfer function of the CCD [8] [10]. The amplitude response of the CCD on a small sinusoidal input signal superimposed on the bias charge is measured in the frequency domain and the small-signal CTI (SCTI) can be determined accurately by curve fitting of the theoretical transfer function to the experimental data. This small-signal approach has been followed incidentally [5], [8], [10] to verify the theoretical expression of the transfer function, but has not been used hitherto to study the SCTI.

For the SCTI, two parameters are important: the bias charge and the transfer time, that is the time available for charge trans. fer. Due to its small-signal nature, our approach is very apt to study the dependence of the SCTI on the bias charge, while the transfer time dependence can be investigated by using a pseudo one-phase clocking scheme, which allows one to vary the transfer time over a large range with a fixed clock frequency.

Experiments have been performed on different types of devices with channel widths ranging from 15 to $300 \mu \mathrm{m}$. Because no dependence of the SCTI on the channel width was found for channel widths larger than $50 \mu \mathrm{m}$, the SCTI originates from interface states in the central gate area, contrary to the predictions on the basis of the edge effect. However, from a numerical calculation of the SCTI due to interface states situated in the central gate region, a large disagreement between the calculated and the experimental SCTI followed. This discrepancy could be solved by applying the so-called McWhorter model [11] for the interface states.

In contrast to the conventional model of interface states, where a unique capture cross section is associated with each energy level, in the McWhorter model a spectrum of capture cross sections is associated with each energy. This model has received a lot of attention for the explanation of $1 / f$ noise in MOS transistors [12], [13]. Using the McWhorter model for the interface states, the experimentally observed behavior of the SCTI can be fully understood.

The organization of this paper is as follows: in Section II an expression for the SCTI is derived, using the McWhorter interface states model. This is followed in Section III by a description of the experimental procedure. In Section IV experimental results are given, in Section $\mathrm{V}$ a discussion of the results is presented, and finally, in Section VI, some conclusions are drawn.

\section{Theory of the Small-Signal Charge TRANSFER INEFFICIENCY}

In a SCCD, operated with a bias charge $Q_{b}$, the CTI is a nonlinear function of the bias charge [5]. The SCTI, which expresses the CTI associated with a small modulation superimposed on $Q_{b}$, is defined by [14]

$$
\epsilon\left(Q_{b}, t^{\prime}\right)=\frac{d Q_{r}\left(t^{\prime}\right)}{d Q_{b}}
$$

with $Q_{r}\left(t^{\prime}\right)$ the remaining charge below the gate after charge transfer during the time interval $\left[0, t^{\prime}\right]$. The transfer time $t^{\prime}$ is explicitly incorporated in (1), because it is an independent parameter in the experimental setup that can be changed by adjusting the width of a transfer pulse (see Section III). $Q_{r}\left(t^{\prime}\right)$ consists of mobile charge and charge captured in interface states, the former being described by the continuity-equation and the latter by the Shockley-Read-Hall (S-R-H) equation. Both equations represent a system of coupled differential equations that cannot be solved analytically. However, even from simplified calculations it is clear that for short transfer times $t^{\prime}, Q_{r}\left(t^{\prime}\right)$ is dominated by the remaining mobile charge, while for long transfer times the captured charge becomes dominant. For the range of transfer times ( $400 \mathrm{~ns}-20 \mu \mathrm{s})$ that is used in this paper, it has been verified with the help of the charge control theory [15] that the amount of remaining mobile charge is negligible and, accordingly, $Q_{r}\left(t^{\prime}\right)$ may be identified as captured charge. Taking into account the influence of the gate length, the above-mentioned range is in agreement with the findings in [1] - [4]. Also experimentally, we have observed for devices with gate lengths $L=20 \mu \mathrm{m}$ an increase in $\epsilon\left(Q_{b}, t^{\prime}\right)$ for transfer times $<400 \mathrm{~ns}$, which could be attributed to incomplete transfer of mobile charge.

Using the McWhorter interface state model, the depth into the oxide to which charges are captured depends on the amount of stored charge. This is visualized by the capture line, which will be explained later on. As a consequence, the modulation superimposed on $Q_{b}$ modulates the position of the capture line as well. This process indicates, that more charges are captured from a larger charge packet, which contributes to the SCTI. However, not all captured charge in such an incremen. tal oxide volume is relevant. For the SCTI, only that captured charge is relevant which is emitted into the next charge packet within the clock period $T_{c}$ following the charge transfer. By definition, this charge is given by the difference of the occupation function [16] at times $t^{\prime}$ and $t^{\prime}+T_{c}$.

Another contribution to the SCTI may be expected from the variation in the amount of charge that remains captured in those states in the oxide that are able to capture charge every clock period. This amount is determined by the occupation function at $t^{\prime}$. However, as $\epsilon\left(Q_{b}, t^{\prime}\right)$ is defined as the derivative of $Q_{r}\left(t^{\prime}\right)$ with respect to $Q_{b}$, the zero level of $Q_{r}\left(t^{\prime}\right)$ can be chosen arbitrarily. From the occupation function during the emission process, which will be derived later on, it is clear that the interface states occupancy at time $t^{\prime}+T_{c}$ is practically independent of $Q_{b}$; hence, it may be subtracted in the definition of $Q_{r}\left(t^{\prime}\right)$. The above discussion is summarized in the following definition of $Q_{r}\left(t^{\prime}\right)$ :

$$
\begin{aligned}
Q_{r}\left(t^{\prime}\right)= & q A\left(Q_{b}\right) \int_{0}^{d} \int_{E_{\mathrm{gap}}} D_{\mathrm{ox}}(E, x) \\
& \cdot\left[g\left(E, t^{\prime}, x\right)-g\left(E, t^{\prime}+T_{c}, x\right)\right] d E d x
\end{aligned}
$$

where $q$ is the unit charge, $A\left(Q_{b}\right)$ the area covered by the bias charge, $d$ is the oxide thickness and $D_{\mathrm{ox}}(E, x)\left(\mathrm{eV}^{-1} \cdot \mathrm{cm}^{-3}\right)$ is the volume concentration of interface states at energy $E$ and distance $x$ from the $\mathrm{Si}-\mathrm{SiO}_{2}$ interface in the oxide. $A\left(Q_{b}\right)$ is a function of the bias charge due to the finite slopes of the poten. 
tial well that contains $Q_{b}$ [7], [17]. The position-dependent occupation function $g(E, t, x)$ at times $t=t^{\prime}$ and at $t=t^{\prime}+T_{c}$ follows from the S-R-H equation, with appropriate boundary conditions. The reasoning which led to the definition of $Q_{r}\left(t^{\prime}\right)$ expresses a signal size effect. Although the underlying physical mechanism is completely different, it resembles the well-known edge effect [2], [3]: for larger charge packets, the captire of charge carriers occurs in a larger $\mathrm{SiO}_{2}$ volume.

Calculating $\epsilon\left(Q_{b}, t^{\prime}\right)$ from $Q_{r}\left(t^{\prime}\right)$ as defined in (2) yields two separate terms: a term expressing the contribution of the edge regions proportional to $d A\left(Q_{b}\right) / d Q_{b}$, and a term proportional to $A\left(Q_{b}\right)$ describing the influence of interface states in the central gate region

$$
\begin{aligned}
\epsilon\left(Q_{b}, t^{\prime}\right)= & q \frac{d A\left(Q_{b}\right)}{d Q_{b}} \int_{0}^{d} \int_{E_{\text {gap }}} D_{\mathrm{ox}}(E, x) \\
& \cdot\left[g\left(E, t^{\prime}, x\right)-g\left(E, t^{\prime}+T_{c}, x\right)\right] d E d x \\
& +q A\left(Q_{b}\right) \int_{0}^{d} \int_{E_{\text {gap }}} D_{\mathrm{ox}}(E, x) \\
& \frac{d}{d Q_{b}}\left[g\left(E, t^{\prime}, x\right)-g\left(E, t^{\prime}+T_{c}, x\right)\right] d E d x .
\end{aligned}
$$

Using the occupation function $g(E, t, x)$ which will be derived later, both terms of this equation can be evaluated, provided $d A\left(Q_{b}\right) / d Q_{b}$ and $D_{\text {ox }}(E, x)$ are known.

The edge term (the first right-hand term in (3)) comjorises two separate contributions originating from interface :tates situated at the parallel and perpendicular edges [3]. As will be discussed in Section $\mathrm{V}$, we did not observe a contribution of the perpendicular edge term to $\epsilon\left(Q_{b}, t^{\prime}\right)$ and, consequently, only the parallel edge term, to be referred to as "edge ternn" in the following, will be considered.

In (3), the central gate term does not depend on the chinnel width $W$, while the edge term is inversely proportional to $W$ [3]. As experiments revealed an $\epsilon\left(Q_{b}, t^{\prime}\right)$ independent on the channel width for $W \geqslant 50 \mu \mathrm{m}$ while only a weak depencience was found for smaller channel widths, we concluded that $\epsilon\left(Q_{b}, t^{\prime}\right)$ originates from interface states in the central gate area. Therefore, we will concentrate on the evaluation of the second term in (3).

To do this, it is first noticed that the application of the McWhorter model yields a position-dependent S-R-H "quation, because the capture cross section now depends on $x$. By calculating the decay of the electron wave function in the $\mathrm{SiO}_{2}$, Heiman and Warfield [18] derived

$$
\sigma_{n}(E, x)=\sigma_{n}(E) e^{-x / x_{0}}
$$

with $\sigma_{n}(E)$ the energy-dependent capture cross section at the $\mathrm{Si}-\mathrm{SiO}_{2}$ interface. The penetration depth $x_{0}$ associated with the electron wave function in the oxide was estimated to be approximately $1 \AA[18]$. Inserting (4) in the S-R-H equation yields

$$
\begin{aligned}
\frac{d g(E, t, x)}{d t}= & \sigma_{n}(E) v_{t h} e^{-x / x_{0}}[1-g(E, t, x)] n_{s}(t) \\
& -\sigma_{n}(E) v_{t h} N_{c} e^{-x / x_{0}} e^{-E / k T} g(E, t, x)
\end{aligned}
$$

with $v_{t h}$ the thermal velocity, $n_{s}(t)$ the volume density of mobile charge carriers, $N_{c}$ the effective density of states in the conduction band, and $k T$ the thermal voltage. $E$ is the energy distance from the conduction band. In (5), the first term on the right-hand side describes the capture of mobile charge carriers, while the second term accounts for the emission process. As the actual trap density $D_{\mathrm{ox}}(E, x)$ and capture cross section $\sigma_{n}(E)$ are not known, a uniform trap density $D_{\mathrm{ox}}\left(\mathrm{eV}^{-1} \cdot \mathrm{cm}^{-3}\right)$ will be assumed, as well as a constant capture cross section $\sigma_{n}$ at the interface. Solutions of (5) corresponding to the storage and the transfer mode in a CCD will be investigated now.

First, the storage mode will be considered. Assume all traps to be empty for $t \leqslant 0$, and assume a mobile charge density $n_{s}$ to be present below the gate for $t>0$. The solution of (5) is then given by

$$
g_{c}(E, t, x)=F\left(E, n_{s}\right)\left[1-e^{-t / \tau_{c}(E, x)}\right]
$$

where the subscript " $c$ " denotes that capture is relevant here. In (6)

$$
F\left(E, n_{s}\right)=\left[1+\frac{N_{c}}{n_{s}} e^{-E / k T}\right]^{-1}
$$

and

$$
\tau_{c}(E, x)=\left[\sigma_{n} v_{t h} e^{-x / x_{0}}\left[n_{s}+N_{c} e^{-E / k T}\right]\right]^{-1} .
$$

$F\left(E, n_{s}\right)$ is a Fermi-Dirac function with an associated quasiFermi level $E_{Q}$

$$
E_{Q}=k T \ln \frac{N_{c}}{n_{s}} .
$$

Because $F\left(E, n_{s}\right)$ is a steep function of energy and $F\left(E_{Q}, n_{s}\right)=$ 0.5 , the quasi-Fermi level $E_{Q}$ can be considered as a separation between states that eventually capture an electron and those that remain empty. From (8), it is seen that the capture time constant $\tau_{c}(E, x)$ depends exponentially on $x$. Furthermore, this time constant enters the exponential factor in (6). Consequently, the expression $\left[1-\exp \left(-t / \tau_{c}(E, x)\right)\right]$ is a very steep function of the position $x$ and approaches a unit step function, which is either 1 or 0 . It behaves similar to a Fermi-Dirac function. This notion led to the definition of a capture line $x_{c}(t)$ [19] which indicates the position of the boundary between full and empty traps. Solving $g_{c}(E, t, x)=0.5$ yields

$$
x_{c}(t)=x_{0} \ln \left[\sigma_{n} v_{t h} n_{s} t\right] .
$$

The capture process can now be visualized as shown in Fig. 1 . The traps are uniformly distributed and indicated by open or black dots, corresponding to empty or filled states, respectively. As the capture continues, the capture line $x_{c}(t)$ moves to the left. Because the charge is stored during half a clock period, at the end of the storage mode, the full traps obey

$$
x \leqslant x_{c}\left(T_{c} / 2\right)
$$

and

$$
E \geqslant E_{Q}
$$

Note from (9) and (10) that both $E_{Q}$ and $x_{c}\left(T_{c} / 2\right)$ depend on the bias charge carrier density $n_{s}$. 


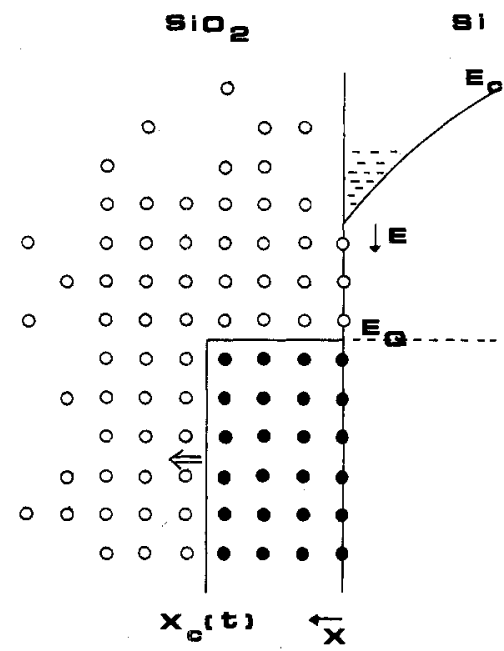

Fig. 1. Schematical representation of the capture process. Black dots indicate filled interface states. If the capture process continues, the capture line shifts to the left.

The emission process can be treated in a similar way. Assuming an ideal transfer of mobile charge (that is $n_{s}=0$ for $t>0$ ) at $t=0$ and taking the start condition for the trap occupation to correspond with $g_{c}\left(E, T_{c} / 2, x\right)$, it follows from (5)

$$
\begin{aligned}
g_{e}(E, t, x)= & g_{c}\left(E, T_{c} / 2, x\right) \\
& \cdot \exp \left(-\sigma_{n} v_{t h} N_{c} t e^{-x / x_{0}} e^{-E / k T}\right)
\end{aligned}
$$

where the subscript " $e$ " denotes the emission process. In (12) the quantities $x / x_{0}$ and $E / k T$ play the same role: both enter the expression in a double exponential way. Hence, they discriminate between filled and empty states, which may be visualized by the emission line $x_{e}(t, E)$ [19]. The emission line follows from solving $g_{e}(E, t, x)=0.5$, with $g_{c}\left(E, T_{c} / 2, x\right)=1$ and is given by the collection of points, obeying

$$
\frac{E}{k T}+\frac{x}{x_{0}}=\ln \left[\sigma_{n} v_{t h} N_{c} t\right] .
$$

Taking into account the initial occupation, the traps that remain occupied by an electron after emission during the time interval $\left[0, t^{\prime}\right]$ are characterized by

$$
\begin{aligned}
& x \leqslant x_{c}\left(T_{c} / 2\right) \\
& E \geqslant E_{Q}
\end{aligned}
$$

and

$$
\frac{E}{k T}+\frac{x}{x_{0}} \geqslant \ln \left[\sigma_{n} v_{t h} N_{c} t^{\prime}\right]
$$

In Fig. 2 the emission process is indicated schematically. The area between the emission lines $x_{e}\left(t^{\prime}, E\right), x_{e}\left(t^{\prime}+T_{c}, E\right)$ and the lines $E=E_{Q}, x=0$, and $x=x_{c}\left(T_{c} / 2\right)$ is a measure for the number of the traps that emit their charge in the time interval $\left[t^{\prime}, t^{\prime}+T_{c}\right]$. This area, indicated by means of the half-filled circles, corresponds to the integrals over the energy and position in (2).

The approximations that have been used in the equations derived above may seem rather crude. It has been verified,

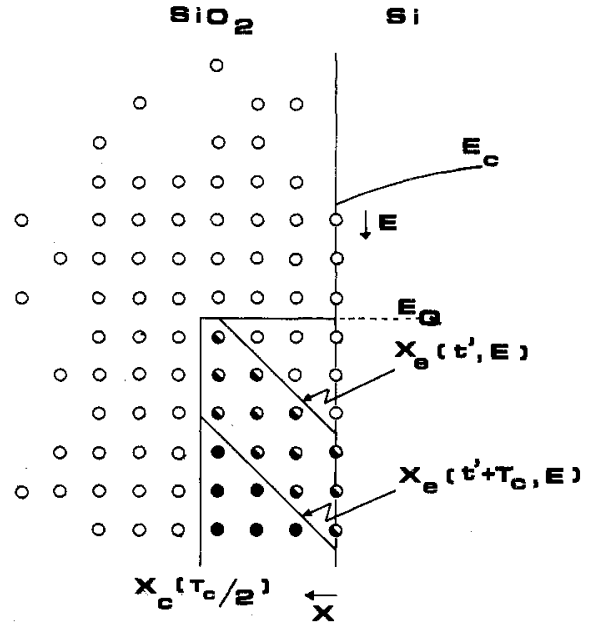

Fig. 2. Schematical representation of the emission process. The emission line $x_{e}\left(t^{\prime}, E\right)$ separates filled and empty states. The capture line $x_{c}\left(T_{c} / 2\right)$ and quasi-Fermi level $E_{Q}$ denote the trap occupation at the end of the storage period.

however, by means of numerical calculations, that all the expressions are fairly good approximations, proving that the very steep transitions in the double exponential functions in (6) and (12) indeed allow the step function approximation.

Having discussed the behavior of the occupation function, we will now return to the calculation of $\epsilon\left(Q_{b}, t^{\prime}\right)$. Using (9), (10), and (13), the above-mentioned area, and hence $Q_{r}\left(t^{\prime}\right)$, can be calculated. In order to obtain the derivative with respect to $Q_{b}$, a relation between $Q_{b}$ and $n_{s}$ is required. Here a linear relationship is used, which is certainly correct for small surface charge density as follows from [20]. It is now a straightforward matter to calculate $\epsilon\left(Q_{b}, t^{\prime}\right)$

$$
\epsilon\left(Q_{b}, t^{\prime}\right)=-q k T \frac{A\left(Q_{b}\right)}{Q_{b}} x_{0} D_{\mathrm{ox}} \ln \frac{t^{\prime}}{t^{\prime}+T_{c}} .
$$

This is our final result: it expresses the SCTI due to interface states situated in the central gate area and represented by the McWhorter interface states model. It predicts a logarithmic dependence of $\epsilon\left(Q_{b}, t^{\prime}\right)$ with increasing transfer time $t^{\prime}$ and an inversely proportional dependence on the bias charge den. sity $Q_{b} / A\left(Q_{b}\right)$.

\section{Experimental Procedure}

In this section, a general description of the devices and the clocking scheme is given followed by a discussion of the experimental setup for measurement of the transfer function and the procedure to extract $\epsilon\left(Q_{b}, t^{\prime}\right)$ from these data.

In Fig. 3, a hybrid picture of a CCD cross section in the direction of charge transfer together with the surface potential is given. A fill-and-spill input was used, and an on-chip source follower was implemented at the output. The bias charge $Q_{b}$ and the modulation are realized by the voltages $V_{b}$ and $v_{\text {in }}$, respectively. The modulation was small, corresponding to a surface charge density modulation less than $5 \times 10^{9} \mathrm{~cm}^{-2}$. The bias charge was varied over a range corresponding from $10^{10}$ to $3 \times 10^{11} \mathrm{~cm}^{-2}$. The clocking scheme is indicated in Fig. 4. The transfer gates $\phi_{t r, 1}$ and $\phi_{t r, 2}$ control the charge 


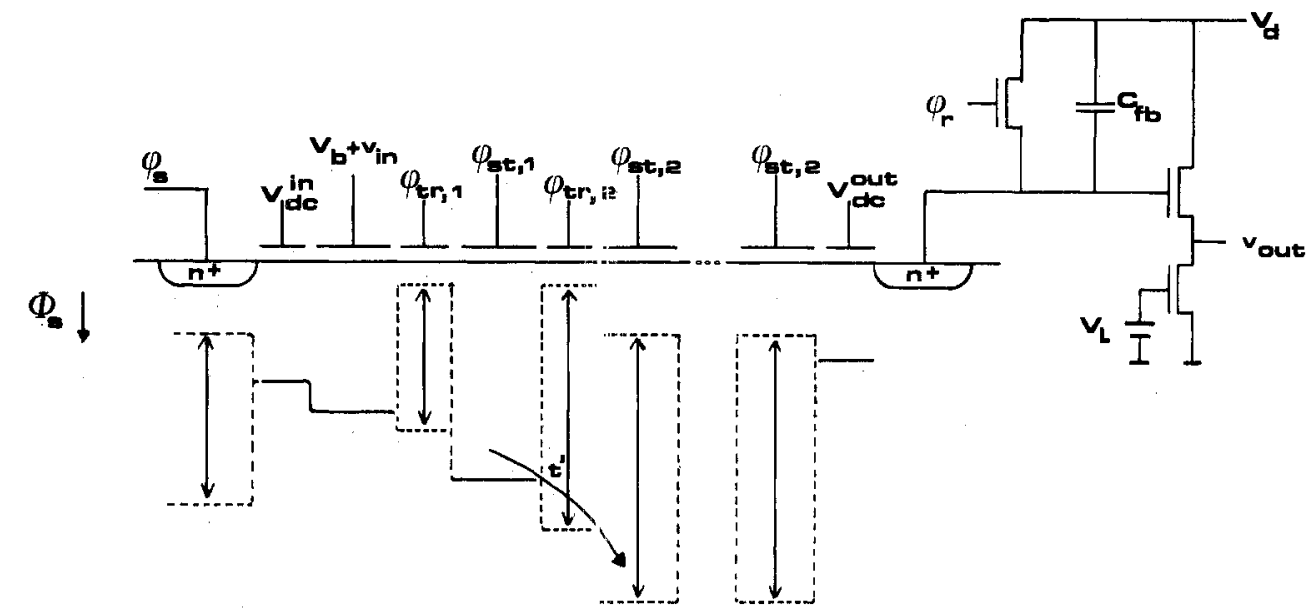

Fig. 3. Hybrid picture of a CCD cross section and assosiated surface potential. The arrow indicates the charge flow during $\left[0, t^{\prime}\right]$.

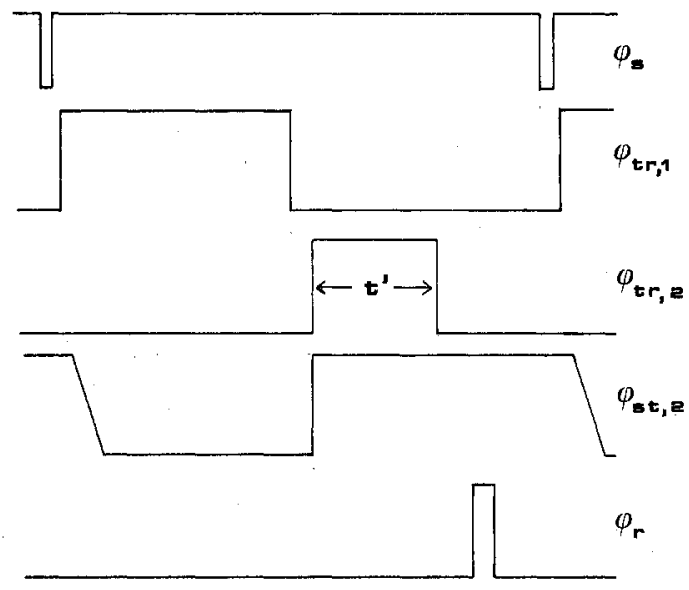

Fig. 4. Representation of the pseudo one-phase clocking scheme. The width of $\phi_{t r, 2}$ determines the transfer time $t^{\prime}$.

transfer between the storage gates $\phi_{s t, 1}$ and $\phi_{s t, 2}$. With the help of Figs. 3 and 4, the operation of the devices can eisily be understood. Note that the width $t^{\prime}$ of the transfer pulse $\phi_{t r, 2}$ determines the time available for charge transfer betv'een the dc storage gate $\phi_{s t, 1}$ and the pulsed storage gate $\phi_{s t, 2}$.

Fig. 5 gives a block scheme of the computer-controlled neasurement setup. The use of a sensitive lock-in amplifier allows accurate determination of the transfer function. Curve fitting of the theoretical transfer function [9] to these data then yields $\epsilon\left(Q_{b}, t^{\prime}\right)$ accurately. The SCTI found in this way represents an average value of the SCTI's associated with the transfers from $\phi_{s t, 1}$ to $\phi_{s t, 2}$ and from $\phi_{s t, 2}$ to $\phi_{s t, 1}$. However, a change in the transfer time $t^{\prime}$ affects only the transfer from $\phi_{s t}$, i to $\phi_{s t, 2}$. Hence, it is possible to calculate the SCTI associated with the latter transfer from the change in the average valus.

\section{EXPERIMENTAL RESULTS}

Experiments have been performed on three different $t$, $p e s$ of devices, indicated by RGCCD, DCCD, and WCCD. The devices were n-channel SCCD's, grown on $\langle 100\rangle \mathrm{Si}$, with a substrate doping $N_{a}=8 \times 10^{14} \mathrm{~cm}^{-3}$, fabricated in an civerlapping two-level polysilicon process. Gate oxide thickness was

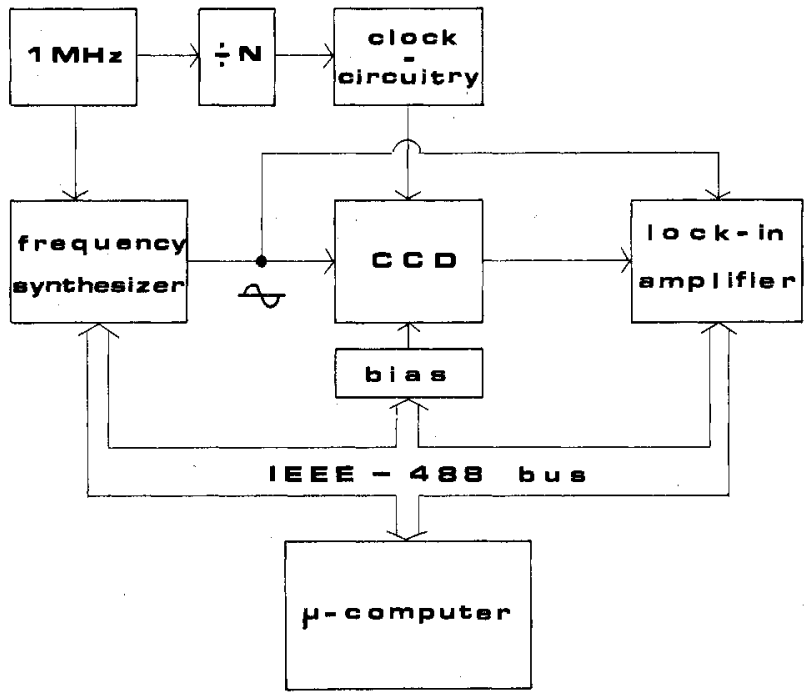

Fig. 5. Block diagram of the experimental setup.

$1000 \AA$. A single CCD delay line was implemented on the RGCCD chips, while on the DCCD- and WCCD-chips, two and three delay lines, respectively, were implemented, which differ in channel width but are identical in all other aspects. Parameters concerning the geometry of these devices are listed in Table I. As expected to be, the quality and nature of the $\mathrm{Si} / \mathrm{SiO}_{2}$ transition region are sensitive to the oxidation and annealing steps, and these will be described in some detail. For the RGCCD, the oxides were grown at $1150^{\circ} \mathrm{C}$ in dry $\mathrm{O}_{2}$ with subsequent downramping with $25^{\circ} \mathrm{C} / \mathrm{min}$ in $\mathrm{N}_{2}$. The second polysilicon layer was oxidized at $1050^{\circ} \mathrm{C}$ for 30 min in dry $\mathrm{O}_{2}$, followed by $30 \mathrm{~min}$ at $1000^{\circ} \mathrm{C}$ in $\mathrm{N}_{2}$. Flowglass was deposited and kept for $40 \mathrm{~min}$ at $1100^{\circ} \mathrm{C}$. An anneal step at $500^{\circ} \mathrm{C}$ in wet $\mathrm{N}_{2}$ for $30 \mathrm{~min}$ before metallization completed the process. The DCCD devices were manufactured in approximately the same process. Here, the second gate oxide was grown at $1050^{\circ} \mathrm{C}$. For the WCCD devices, ion implantation was used to define the active area and source and drain; hence, less high-temperature steps were necessary. Here, both oxides were grown at $1050^{\circ} \mathrm{C}$ in dry $\mathrm{O}_{2}$ with subsequent downramping 
TABLE I

Geometrical Parameters of the CCD Delay Lines

\begin{tabular}{|c|c|c|c|}
\hline & RGCCD & WCCD & DCCD \\
\hline $\begin{array}{l}\text { channel width } \\
\text { storage gate }\end{array}$ & $390 \mathrm{\mu m}$ & $50,150,300 \mu \mathrm{m}$ & $15,300 \mu \mathrm{m}$ \\
\hline $\begin{array}{l}\text { length } \\
\text { transfer gate }\end{array}$ & $2 \varnothing \mu \mathrm{m}$ & $12 \mu \mathrm{m}$ & $12 \mu \mathrm{m}$ \\
\hline $\begin{array}{l}\text { length } \\
\text { number of delay } \\
\text { sections }\end{array}$ & $\begin{array}{l}10 \mu \mathrm{m} \\
26\end{array}$ & $\begin{array}{l}8 \mu m \\
24\end{array}$ & $\begin{array}{l}8 \mu \mathrm{m} \\
24,74\end{array}$ \\
\hline
\end{tabular}

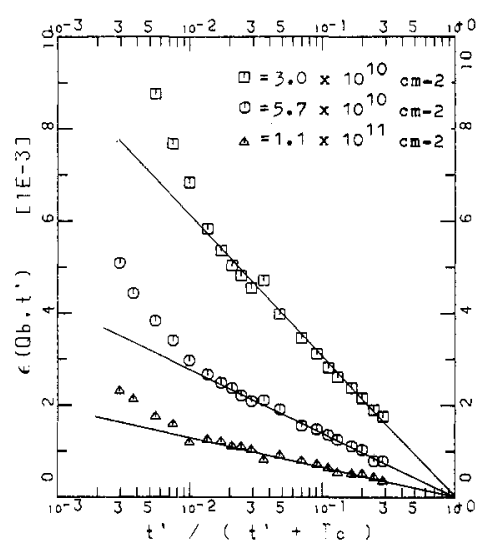

Fig. 6. $\epsilon\left(Q_{b}, t^{\prime}\right)$ as a function of the transfer time with the bias charge density as parameter. Clock frequency $F_{c}=25 \mathrm{kHz}$, device RGCCD 820508 .

in $\mathrm{N}_{2}$. Implantation of source and drain was followed by a 60 -min $1000^{\circ} \mathrm{C}$ anneal in $\mathrm{N}_{2}$, and oxidation of the second polysilicon layer at $1050^{\circ} \mathrm{C}$ for $30 \mathrm{~min}$ in dry $\mathrm{O}_{2}$. The same anneal step before metallization as in the RGCCD process was used.

Now experimental data are presented in order to verify the theory developed in Section II. First, in Fig. 6 the dependence on the transfer time is illustrated by means of a plot of $\epsilon\left(Q_{b}, t^{\prime}\right)$ versus $\ln \left(t^{\prime} /\left(t^{\prime}+T_{c}\right)\right)$, with the bias charge density as a parameter. The solid lines, calculated according to (15), describe the experimental points well. For transfer times $t^{\prime}<400 \mathrm{~ns}$ (that is $\left.t^{\prime} /\left(t^{\prime}+T_{c}\right)<0.01\right)$ a larger $\epsilon\left(Q_{b}, t^{\prime}\right)$ is found than expected on basis of (15). This indicates the onset of the incomplete transfer of mobile charge due to the relatively long gate length $(20 \mu \mathrm{m})$. In the WCCD and DCCD devices, the incomplete transfer of mobile charge becomes only manifest for $t^{\prime}<100 \mathrm{~ns}$. It was verified that the $\ln \left(t^{\prime} /\left(t^{\prime}+T_{c}\right)\right)$ dependence holds for different clock periods $T_{c}$.

To validate the dependence on the bias charge density as predicted by (15), the slope of $\epsilon\left(Q_{b}, t^{\prime}\right)$ with respect to $\ln \left(t^{\prime}\right)$ $\left(t^{\prime}+T_{c}\right)$ ) was determined as a function of the bias charge. This was done by means of linear regression using values of $\epsilon\left(Q_{b}, t^{\prime}\right)$ measured for different transfer times. This procedure was applied to data obtained from the RGCCD devices with clock frequency $F_{c}=25 \mathrm{kHz}$ and for the WCCD devices with $F_{c}=100 \mathrm{kHz}$. In Fig. 7 the results are plotted versus the inverse bias charge density. The predicted inverse proportion.

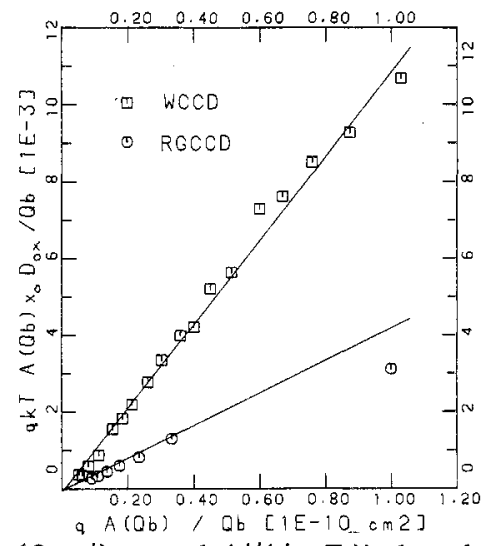

Fig. 7. Slope of $\epsilon\left(Q_{b}, t^{\prime}\right)$ versus $\ln \left(t^{\prime} /\left(t^{\prime}+T_{c}\right)\right)$ plotted as a function of the inverse bias charge density. Clock frequency $F_{c}=100 \mathrm{kHz}$, device WCCD 830906, $W=150 \mu \mathrm{m}$, and $F_{c}=25 \mathrm{kHz}$, device RGCCD 820508 , respectively.

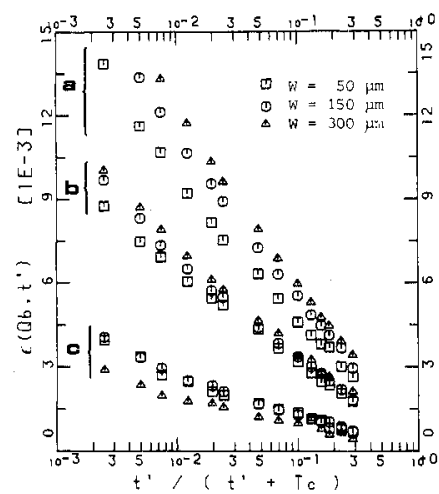

Fig. 8. $\epsilon\left(Q_{b}, t^{\prime}\right)$ as a function of the transfer time with the bias charge density as parameter for several channel widths. The bias charge densities for the data labeled a, b, and $\mathrm{c}$ are $4.2 \times 10^{10} \mathrm{~cm}^{-2}, 6.2 \times 10^{10}$ $\mathrm{cm}^{-2}$, and $1.2 \times 10^{11} \mathrm{~cm}^{-2}$, respectively. Clock frequency $F_{c}=$ $25 \mathrm{kHz}$, device WCCD 830903.

ality with respect to the bias charge density is present indeed. The quantity $x_{0} D_{\text {ox }}$ can be determined from the data in Fig. 7 and was found to be $4.3 \times 10^{9} \mathrm{eV}^{-1} \cdot \mathrm{cm}^{-2}$ for the WCCD series and $1.6 \times 10^{9} \mathrm{eV}^{-1} \cdot \mathrm{cm}^{-2}$ for the RGCCD series.

In Fig. 8, $\epsilon\left(Q_{b}, t^{\prime}\right)$ is plotted similarly as in Fig. 6 for the WCCD devices with channel widths $W=50,150$, and $300 \mu \mathrm{m}$. Each triplet of lines corresponds to the same bias charge density and within a triplet each line corresponds to a different $W$. The small differences in $\epsilon\left(Q_{b}, t^{\prime}\right)$ within each triplet are due to minor differences in the operational conditions of the devices and are not significant. The data in this figure show that for these channel widths $\in\left(Q_{b}, t^{\prime}\right)$ does not depend on $W$, which proves that the edge effect is not important here.

It may be anticipated, however, that a contribution of traps in the edge region will become more pronounced for devices with a small $W$. This was verified by means of the CCD's with widely differing channel widths that were implemented on the DCCD devices. The transfer time dependence of the edge term, as expressed in (3), can be calculated from numerical integration, and was found to obey approximately the same loga. rithmic law as the central gate term. Fig. 9 illustrates these remarks: here, $\epsilon\left(Q_{b}, t^{\prime}\right)$ obtained from the DCCD devices is 


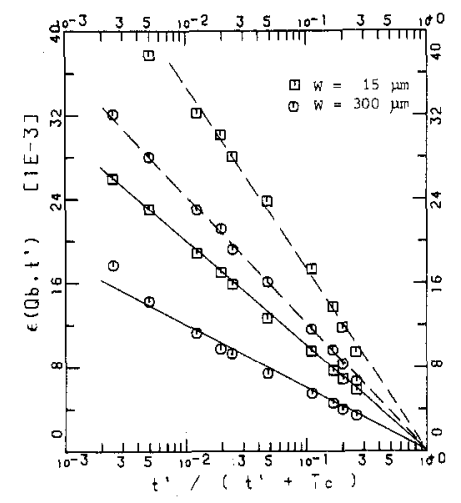

Fig. 9. $\epsilon\left(Q_{b}, t^{\prime}\right)$ as a function of the transfer time with the bias clarge density as a parameter, for two widely differing channel widths. Solid and broken lines denote identical bias charge densities, $2.8 \times 10^{11}$ $\mathrm{cm}^{-2}$ and $1.5 \times 10^{11} \mathrm{~cm}^{-2}$ respectively. Device DCCD 830417, $F_{c}=$ $25 \mathrm{kHz}$.

plotted similarly as in Figs. 6 and 8 for $W=15$ and $300 \mu \mathrm{m}$. The $W=15-\mu \mathrm{m}$ device exhibits a SCTI which is twice as large as found for the $300-\mu \mathrm{m}$ device due to the increasing importance of the edge effect for smaller channel widths.

\section{Discussion}

It was stated in the Introduction that the conventional interface state model with a single capture cross section at rach energy level cannot account for the experimentally obse:ved SCTI. As this statement is vital for our interpretation, it will be elaborated here. Using the conventional interface state model, Mohsen et al. [3] already pointed out that the CTI due to interface states situated in the central gate area approaches zero for sufficiently large transfer time and bias charge. liy a numerical calculation of the SCTI based on the conventional interface state model we confirmed this conclusion. In o:der to perform these calculations, the SCTI due to interface states in the central gate area was defined similar as in (3)

$$
\begin{aligned}
& \epsilon_{\mathrm{con}}\left(Q_{b}, t^{\prime}\right)=q A\left(Q_{b}\right) D_{i t} \\
& \quad \cdot \int_{E_{\text {gap }}} \frac{d}{d Q_{b}}\left[f\left(E, t^{\prime}\right)-f\left(E, t^{\prime}+T_{c}\right)\right] d E
\end{aligned}
$$

with $D_{i t}$ the density of interface states $\left(\mathrm{eV}^{-1} \cdot \mathrm{cm}^{-2}\right)$ and the subscript "con" to denote the conventional interface state model. The occupation function $f(E, t)$ was computed f:om the numerical solution of the S-R-H equation taking nto account the effect of decrease of mobile charge, which was modeled according to [15]. $\epsilon_{\mathrm{con}}\left(Q_{b}, t^{\prime}\right)$ can now be computed as a function of the transfer time. In Fig. 10 the results of this procedure are shown together with a typical set of ex jerimental data. The dotted curve was calculated using $D_{t}=$ $2 \times 10^{10} \mathrm{eV}^{-1} \cdot \mathrm{cm}^{-2}$ and $\sigma_{n}=2 \times 10^{-15} \mathrm{~cm}^{-2}$, obtained from charge pumping measurements [21] on an on-chip lest MOST, while the solid curve was computed using $D_{i t}=2 \mathrm{X}$ $10^{11} \mathrm{eV}^{-1} \cdot \mathrm{cm}^{-2}$. Neither the transfer time dependence nor the magnitude of the calculated SCTI is in accordance vith the experimental points. Note that the calculated SCTI approaches zero for long transfer times. It has been veri.ied that this conclusion holds for all reasonable choices of the

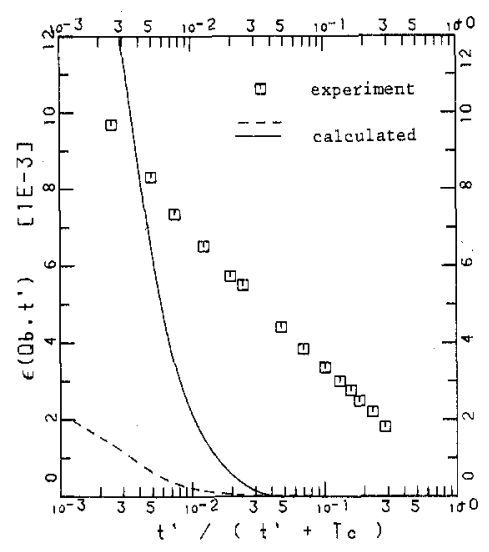

Fig. 10. Comparison of the calculated $\epsilon\left(Q_{b}, t^{\prime}\right)$ on the basis of the conventional interface state model and experimental data all corresponding to the bias charge density $6.2 \times 10^{10} \mathrm{~cm}^{-2}$. Dotted curve calculated with $D_{i t}=2 \times 10^{10} \mathrm{eV}^{-1} \cdot \mathrm{cm}^{-2}$ and $\sigma_{n}=2 \times 10^{-15} \mathrm{~cm}^{2}$ solid curve with $D_{i t}=2 \times 10^{11} \mathrm{eV}^{-1} \cdot \mathrm{cm}^{-2}$ and same $\sigma_{n}$. Experimental data obtained from WCCD 830903, $W=150 \mu \mathrm{m}$.

capture cross section and other relevant parameters used in the numerical calculation.

The model presented by Klar et al. [6] predicts a major contribution to $\epsilon\left(Q_{b}, t^{\prime}\right)$ of interface states situated at the perpendicular edge with a magnitude proportional to $\Delta L / L$, where $\Delta L$ is the "length" of the perpendicular edge region. Now $\Delta L$ is determined by the slope of the potential well, which in turn is determined by the fringing fields near the overlap between storage gate and adjacent transfer gates. Varying the base-line voltages of $\phi_{t r, 1}$ and $\phi_{t r, 2}$, respectively, (see Fig. 3), we were able to change $\Delta L$ experimentally. No sig. nificant dependence of $\epsilon\left(Q_{b}, t^{\prime}\right)$ on the magnitude variations of the perpendicular edge region induced in this way could be observed, proving the insignificance of the perpendicular edge effect. This observation is in agreement with the conclusions in [3].

From the above it follows that if the conventional interface state model would apply, the nonvanishing contribution of states in the parallel edge region should be dominant for long transfer times. Furthermore, it can be shown that this edge effect yields the same transfer time dependence for the SCTI, as was calculated in (15) for the SCTI due to the McWhorter model interface states in the central gate area. The reason for this similarity was already mentioned: the SCTI in both models is due to a signal size effect and contributing interface states act similarly in both models. This clarifies why the experimentally observed transfer time dependence in the SCTI [22] has been falsely explained in the past by the edge effect, on the basis of the conventional interface state model. However, experiments on several devices with different channel widths did not reveal a proportionality with the inverse channel width, as could be expected in case of a dominant edge effect. Therefore, we have to recant our conclusions stated in [22], where the edge effect was supposed to be responsible for the observed SCTI. We note that our resistive gate experiments in [22] are very well explained by the currently presented model.

It was shown that the SCTI experiments can be satisfactorily explained by means of the McWhorter interface states model. 
A further argument in favor of this model was found by a comparison of the quantity $x_{0} D_{\mathrm{ox}}$ as determined from our measurements with the magnitude of the $1 / f$ noise of on-chip MOS transistors. This was done for devices on the RGCCD, WCCD, and DCCD chips, with $x_{0} D_{\mathrm{ox}}$ equal to $1.6 \times 10^{9}$ $\mathrm{eV}^{-1} \cdot \mathrm{cm}^{-2}, 4.3 \times 10^{9} \mathrm{eV}^{-1} \cdot \mathrm{cm}^{-2}$, and $2.3 \times 10^{10} \mathrm{eV}^{-1}$. $\mathrm{cm}^{-2}$, respectively. It turned out that the magnitude of the $1 / f$ noise is in close agreement with the predictions based on our determination of $x_{0} D_{\text {ox }}$. Details of this work will be published elsewhere [23].

The McWhorter model, or a closely related model [24], has been proposed as an explanation for a number of experiments: $1 / f$ noise [12], [24] and transfer conductance [25] in MOS transistors, conductance measurements [26]-[28], and recently CC-DLTS measurements [19] on MOS capacitors. In relation to conductance measurements, it is known as the Preier model [26]. About the validity of the McWhorter description of interface states, however, no consensus exists [13] , [29].

The essential feature of the McWhorter model is the spectrum of capture cross sections associated with each energy level. Usually, a distribution of traps in the oxide is considered to be the origin, but any other physical mechanism leading to a spectrum of capture cross sections could serve as an explanation as well.

Thus far, experimental evidence concerning the incomplete charge transfer in SCCD's has been obtained solely by the periodic pulse technique as proposed by Broderson [5] or variations thereof [30]. Crucial in this technique is the measurement of the charge loss in the first "ONE" after a number of "ZERO's" has been transferred, as a function of the size of the "ONE" with the magnitude of the "ZERO" as a parameter. According to Broderson et al. [5], the resulting charge loss can be explained by the so-called proportional loss and fixed loss, ascribed to the action of interface states situated at the parallel edges and the central gate area, respectively. However, we found [31] that the results of the periodic pulse technique in the presence of a bias charge can be quantitatively understood according to the presented model by considering the incremental oxide volume in which charges are captured. This might indicate that the concepts of fixed and proportional loss are not adequate to describe the charge loss in CCD's. It is not clear to what extent these findings affect the results obtained by Kriegler et al. [30]. On the one hand, the concept of fixed loss is essential in their experiments; on the other hand, the fixed loss is determined in the absence of bias charge. However, we emphasize that a unique capture cross section at each energy level, as was found by Kriegler et al. [30], is not sufficient to explain the experimentally observed SCTI.

\section{CONCLUSION}

The measurement of the transfer function of CCD's yields very accurate results about the small-signal charge transfer inefficiency. The behavior of the SCTI as a function of the transfer time and bias charge and its very weak dependence on the channel width cannot be understood on the basis of the conventional interface state model. It turns out that the McWhorter model, assuming a uniform distribution of states in the $\mathrm{SiO}_{2}$, perpendicular to the interface provides a complete description of the experimentally observed SCTI. The quantity $x_{0} D_{\mathrm{ox}}$, denoting the penetration depth times the volume density of interface states, determines the SCTI completely.

\section{ACKNOWLEDGMENT}

The authors would like to thank A. Aarnink and J. Holleman for manufacturing the devices and O.W. Memelink for encouraging discussions.

\section{REFERENCES}

[1] J. E. Carnes and W. F. Kosonocky, "Fast-interface-state losses in charge-coupled devices," Appl. Phys. Lett., vol. 20, pp. 261-263, 1972.

[2] M. F. Tompsett, "The quantitative effects of interface states on the performance of charge-coupled devices," IEEE Trans. Electron Devices, vol. ED-20, pp. 45-55, 1973.

[3] A. M. Mohsen, T. C. McGill, Y. Daimon, and C. A. Mead, "The influence of interface states on incomplete charge transfer in overlapping gate charge-coupled devices," IEEE J. Solid-State Circuits, vol. SC-8, pp. 125-138, 1973.

[4] W. F. Kosonocky and J. E. Carnes, "Polysilicon-aluminum gate CCD," in Proc. Charge-Coupled Device Applications Conf., (San Diego, CA), pp. 217-277, 1973.

[5] R. W. Broderson, D. D. Buss, and A. F. Tasch, "Experimental characterization of transfer inefficiency in charge-coupled devices," IEEE Trans. Electron Devices, vol. ED-22, pp. 40-46, 1975.

[6] H. Klar, M. Mauthe, and H. R. Deppe, "Investigations about the edge effect in SCCD's," in Proc. 5th Int. Conf. CCD's, (Edinburgh, Scotland), pp. 408-412, 1979.

[7] J. Y. Chen and C. R. Viswanathan, "An interactive two-dimensional model for designing VLSI CCD's," IEEE Trans. Electron Devices, vol. ED-30, pp. 1135-1142. 1983.

[8] G. F. Vanstone, J. B. G. Roberts, and A. E. Long, "The measurement of the charge residual for CCD transfer using impulse and frequency responses," Solid-State Electron., vol. 17, pp. 889$895,1974$.

[9] H. Wallinga, "A general model for the frequency response of multiphase charge transfer delay lines," IEEE J. Solid-State Circuits, vol. SC-14, pp. 653-655, 1979.

[10] - "Charge-coupled devices for sampled analog signal processing," Ph.D. dissertation, Twente University of Technology, The Netherlands, 1980.

[11] A. L. McWhorter, " $1 / f$ noise and germanium surface properties," in Semiconductor Surface Physics, R. H. Kingston, Ed. Philadelphia: University of Pennsylvania Press, 1957, pp. 207-228.

[12] A. van der Ziel, "Noise in solid-state devices and lasers," Proc. IEEE, vol. 58, pp. 1178-1206, 1970.

[13] F. N. Hooge, T. G. M. Kleinpenning, and L. K. J. Vandamme, "Experimental studies on $1 / f$ noise," Rep. Progr. Phys., vol. 44, pp. 479-532, 1981.

[14] C. N. Berglund and K. K. Thornber, "Incomplete transfer in charge-transfer devices," IEEE J. Solid-State Circuits, vol. SC-8, pp. 108-115, 1973.

[15] H. S. Lee and L. G. Heller, "Charge-control method of chargecoupled device transfer analysis," IEEE Trans. Electron Devices, vol. ED-19, pp. 1270-1279, 1972.

[16] J. G. Simmons and L. S. Wei, "Theory of transient emission current in MOS devices and the direct determination interface trap parameters," Solid-State Electron., vol. 17, pp. 117-124, 1974.

[17] G. F. Amelio, "Computer modeling of charge-coupled device characteristics," Bell Syst. Tech. J., vol. 51, pp. 705-730, 1972.

[18] F. P. Heiman and G. Warfield, "The effect of oxide traps on the MOS capacitance," IEEE Trans. Electron Devices, vol. ED-12, pp. $167-178,1965$.

[19] P. van Staa, H. Rombach, and R. Kassing, "Time-dependent response of interface states in indium phosphide metal-insulatorsemiconductor capacitors investigated with constant-capacitance deep-level transient spectroscopy," J. Appl. Phys., vol. 54, pp. 4014-4021, 1983.

[20] D. G. Ong and R. F. Pierret, "Approximate formula for surface carrier concentration in charge-coupled devices," Electron. Lett., vol. 10 , pp. 6-9, 1974. 
[21] G. Groeseneken, H. E. Maes, N. Beltran, and R. F. de Keersmaecker, "The energy distribution of $\mathrm{Si} / \mathrm{SiO}_{2}$ interface states measured in small size MOSFET's using the charge pumping technique," in Insulating Films on Semiconductors, J. F. Verweij and D. R. Wolters, Eds. Amsterdam: Elsevier, 1983, pp. 153-1:7.

[22] R. G. M. Penning de Vries and H. Wallinga, "Experimental 'erification of increased $D_{i t}$ at the parallel edges of a surface-channel CCD," in Insulating Films on Semiconductors, J. F. Verwe 1 and D. R. Wolters, Eds. Amsterdam: Elsevier, 1983, pp. 171-1;6.

[23] L. K. J. Vandamme and R. G. M. Penning de Vries, "Corre ation between MOST $1 / f$ noise and CCD transfer inefficiency," "iolidState Electron., submitted for publication.

[24] H. S. Fuh and C. T. Sah, "Theory and experiments on surface $1 / f$ noise," IEEE Trans. Electron Devices, vol. ED-19, pp. 273-285, 1972.

[25] J. Koomen, "Interface studies of the MOS structure by transferadmittance measurements," Solid-State Electron., vol. 17 pp.
$321-328,1974$.

[26] H. Preier, "Contributions of surface states to MOS impedance," Appl. Phys. Lett., vol. 10, pp. 361-363, 1967.

[27] D. H. Eaton and C. T. Sah, "Frequency response of $\mathrm{Si}_{-} \mathrm{SiO}_{2}$ interface states on thin oxide MOS capacitors," Phys. Status Solidi $A$, vol. 12, pp. 95-109, 1972.

[28] A. Ushirokawa, M. Warashina, and A. Nagami, "Two-dimensional parameter conductance method for estimation of interface states in MIS structure," Japan J. Appl. Phys., vol. 12, pp. 388-397, 1973.

[29] H. Deuling, E. Klausmann, and A. Goetzberger, "Interface states in $\mathrm{Si}_{-} \mathrm{SiO}_{2}$ interfaces," Solid-State Electron., vol. 15, pp. 559$571,1972$.

[30] R. J. Kriegler, T. F. Devenyi, K. D. Chik, and J. Shappir, "Determination of surface-state parameters from transfer-loss measurements in CCD's," J. Appl. Phys., vol. 50, pp. 398-401, 1979.

[31] R. G. M. Penning de Vries and H. Wallinga, to be published.

\title{
CASTAM: A Process Variation Analysis Simulator for MIOS LSI's
}

\author{
YUKIO AOKI, TORU TOYABE, MEMBER, IEEE, SHOJIRO ASAI, MEMBER, IEEE, AND TAKAAKI HAGIWARA
}

\begin{abstract}
A simulator named CASTAM, which includes both prc cess and device models, has been developed to predict MOS process yariations through the analysis of variations in electrical characteristics of fabricated MOS devices using the Monte Carlo method.

Analysis accuracy using the simulator is examined. Investigation shows that process parameter variations can be estimated with an error of less than 10 percent if an appropriate set of device characteristic items is chosen.

Wafer inspection data for a CMOS pilot line can be analyzed with this simulator, and the main cause of threshold voltage variation pinpointed. Predictions derived from the analyzed results have been confirmed using experimental data. This shows that analysis using CASTAM is sufficiently reliable.
\end{abstract}

\section{INTRODUCTION}

$\mathrm{W}$ ITH THE progress made in semiconductor devices from IC's to VLSI's, the minimum feature length is getting smaller and integration density is becoming higher. Correspondingly, process conditions are becoming more and more complex, and it is becoming very difficult to maintain a high production yield. In the planning of countermeasures to $\mathrm{m}$ prove yield, it is useful to know which process step variation is

Manuscript received March 14, 1984; revised May 7, 1984.

The authors are with Central Research Laboratory, Hitachi I d., Kokubunji, Tokyo 185, Japan. the main cause of variation in device characteristics. It is, however, hard to directly measure variations in process parameters. In 1964, D. P. Kennedy et al. [1] proposed a method by which variations in process parameters could be estimated from measured variations in device characteristics, and they applied this method to bipolar transistors. Recently W. May et al. [2] have reported on almost the same method, and they also analyzed variations in bipolar transistors.

The purpose of the work being reported on here has been to develop CASTAM, a Computer Aided STAtistical Modeling simulator. This program was first applied to MOS devices [3], being different from the above literature.

\section{Anal ytical Method}

An outline of CASTAM is shown in Fig. 1. In a process line that produces LSI's, attempts are made to maintain the same process conditions; however, they unavoidably tend to vary from batch to batch and the device characteristics then scatter around an average value.

The first step in this work using CASTAM is thus to estimate quantitative variations in process parameters that have been difficult to measure directly.

The process line consists of many kinds of steps, and each 